\title{
End-to-End Enforcement of Erasure and Declassification
}

\author{
Stephen Chong Andrew C. Myers \\ Department of Computer Science \\ Cornell University \\ \{schong,andru\}@cs.cornell.edu
}

\begin{abstract}
Declassification occurs when the confidentiality of information is weakened; erasure occurs when the confidentiality of information is strengthened, perhaps to the point of completely removing the information from the system.

This paper shows how to enforce erasure and declassifcation policies. A combination of a type system that controls information flow and a simple runtime mechanism to overwrite data ensures end-to-end enforcement of policies. We prove that well-typed programs satisfy the semantic security condition noninterference according to policy.

We extend the Jif programming language with erasure and declassification enforcement mechanisms and use the resulting language in a large case study of a voting system.
\end{abstract}

\section{Introduction}

Enforcing information security is an important requirement of many systems. However, often information security changes over time, complicating enforcement. Declassification and erasure are two common ways in which the security enforced on information changes. Declassification occurs when the confidentiality enforced on information is weakened, for example, by allowing more people to read the information. Erasure [2] is the opposite phenomenon, occurring when the confidentiality enforced on information is strengthened, perhaps to the point of removing the information from the system entirely.

Much work in recent years has considered how to provide end-to-end enforcement of declassification requirements. (See Sabelfeld and Sands [22] for a recent survey.) Comparatively little work [12] has considered end-to-end

This work was supported by National Science Foundation grant 0430161. The views and conclusions contained herein are those of the authors and should not be interpreted as necessarily representing the official policies or endorsements, either express or implied, of these organizations or the U.S. Government. The U.S. Government is authorized to reproduce and distribute reprints for Governmental purposes notwithstanding any copyright notation thereon. enforcement of erasure requirements, and none has considered both declassification and erasure together. In this paper, we enforce both erasure and declassification requirements end-to-end in a language-based setting. The erasure policies we enforce are significantly more expressive than any previously enforced.

Consider, as an example of erasure requirements, a medical information website. The website offers (among other functionality) a diagnostic application, where a user may enter information about symptoms, and the application will present information about possible diseases consistent with the symptoms. The website's privacy policy states that symptoms the user enters are private, and no record of them will be kept after the user has finished using the diagnostic application. The provider of this website needs to enforce an erasure requirement: when the user has finished using the diagnostic application, the symptom data that the user has entered must be erased. Note that the information the user has entered may need to persist over several user requests, but also might need to be erased before the session has finished. Thus, the lifetime of the information does not necessarily match that of any web server resource. Another subtlety is that the diagnoses the system has produced must also be erased, as the diagnoses may reveal information about the symptoms entered.

Information security is an end-to-end requirement: appropriate security must be enforced on information from when it enters the system until it leaves. Appropriate security should also be enforced on data derived from sensitive information, since the derived data may allow the original information to be deduced. In the diagnostic application described above, learning diagnoses may reveal symptoms, which are sensitive.

Information-flow control is a way to achieve end-to-end enforcement. Information-flow control techniques enforce security by restricting the flow, or propagation, of information in a system. Conceptually, information-flow control techniques label data with security levels; as data are updated and created, the security labels are also updated to reflect data dependencies. The security labels can be used to 
prevent confidential data from being output on public channels. Static approaches [21] have been successful in controlling information flow without incurring the performance overheads of representing the security labels at runtime.

In this paper, we use static information-flow control to enforce erasure and declassification requirements end-toend. Erasure and declassification requirements are specified in a policy language that can express when information may be declassified, and when information must be erased. Section 2 reviews this policy language, which was introduced in earlier work [2] without any enforcement mechanism.

Section 3 presents a simple imperative language that has a runtime mechanism for overwriting memory locations. A type system controls information flow, ensuring that information that needs to be erased is placed only in memory locations that will be overwritten at appropriate times.

Noninterference [7] is an end-to-end semantic security condition. It is well-known that noninterference is too strong in the presence of declassification, which intentionally makes sensitive information public. However, noninterference is too weak in the presence of erasure-it cannot express erasure requirements, which restrict observation of public information. Section 4 proves that well-typed programs satisfy noninterference according to policy [2], a generalization of noninterference that precisely expresses the information flows permitted by declassification and erasure.

Section 5 describes how we incorporated declassification and erasure policies into the decentralized label model [17] and extended the Jif programming language [18] with the new label model. We have used this extended version of Jif to implement a large, security-intensive system: Civitas [4], a secure voting service. Section 6 describes how the policies are useful in its implementation. Section 7 reviews related work, and Section 8 concludes.

\section{Policies}

The declassification and erasure policy framework introduced in previous work [2] assumes there is a lattice $(\mathcal{L}, \sqsubseteq)$ of confidentiality levels, and a language for specifying conditions, which indicate when declassification may occur and when erasure must occur. To instantiate the policy framework, lattice $\mathcal{L}$ and the condition language must be specified. Appropriate security lattices include the two-point lattice $\{L, H\}$ where $L \sqsubseteq H$ and $H \nsubseteq L$, and the lattice of security principals ordered by an acts-for relation [17]. (In Section 5 we use the lattice of security principals when extending the decentralized label model [17].) We assume there is a clear notion of enforcement of confidentiality level $\ell \in \mathcal{L}$ on information. Many condition languages are possible; Section 3 uses program expressions as conditions.
$a, b$
Conditions
$p, q::=$
$\ell$
Policies
$p \searrow q q$

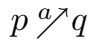
Lattice policy
Declassification policy
Erasure policy

\section{Figure 1. Syntax of policies}

\subsection{Syntax}

Security policies describe what confidentiality level is currently enforced on information, and how this may and must change in the future. Figure 1 shows the syntax of policies. Lattice policy $\ell \in \mathcal{L}$ means that the confidentiality level $\ell$ (or a more restrictive confidentiality level) must be enforced on information now and at all times in the future. Declassification policy $p \searrow^{a} q$ means that policy $p$ is currently enforced on information, and when condition $a$ is satisfied, information may be declassified, after which policy $q$ must be enforced (regardless of the subsequent satisfaction or non-satisfaction of $a$ ). Erasure policy $p$ a $q$ means that policy $p$ is currently enforced on information, and when condition $a$ is satisfied, information must be made more restricted, by enforcing both policies $p$ and $q$ on the information (regardless of the subsequent satisfaction or non-satisfaction of $a$ ).

The satisfaction of conditions controls when declassification may occur, and when erasure must occur. Condition satisfaction is specific to the condition language used. We assume the condition satisfaction depends only on the current system state $s$ (which may include the history of the system), and write $s \vDash a$ if condition $a$ is satisfied in state $s$, and $s \not \models a$ if $a$ is not satisfied in state $s$.

For example, if we are enforcing policy $H \searrow^{a} L$ on information, then we must enforce the confidentiality level $H$ on the information; however, when condition $a$ is satisfied, we are permitted to change the confidentiality level enforced on the information to $L$. If we are enforcing erasure policy $L$ a $H$ on information, then we must enforce the confidentiality level $L$ on the information, and if and when condition $a$ is satisfied, we must change the confidentiality level we are enforcing to be at least as restrictive as both $L$ and $H$-since $L \sqsubseteq H$, it suffices to enforce the confidentiality level $H$.

Consider enforcing policy $\left(H \searrow^{a} L\right) b / H$ on information. Initially policy $H \searrow^{a} L$ is enforced on information, meaning that the confidentiality level $H$ must be enforced, and if condition $a$ is satisfied (before $b$ is satisfied) then confidentiality level $L$ can be enforced on information. However, once condition $b$ is satisfied, we must enforce policy $H$ on information, meaning that confidentiality level $H$ will be enforced then and at all times in the future. 


$$
\frac{\operatorname{reqErase}(p, s)}{\operatorname{reqE} \operatorname{Erase}\left(p \searrow^{a} p^{\prime}, s\right)} \quad \frac{\operatorname{reqE} \operatorname{Erase}(p, s) \text { or } s \vDash a}{\operatorname{reqErase}\left(p^{a} /^{\prime}, s\right)}
$$

Figure 2. Definition of reqErase $(p, s)$

To see how these policies can capture the security requirements of applications, let us revisit the medical information website example from the introduction. A suitable policy for symptoms entered by the user could be session appEnd/T, where session is a confidentiality level allowing only the session client and server to read the information, $T$ is a confidentiality level so restrictive that it prevents the server from storing the information, and appEnd is a condition that is satisfied when the user has finished using the diagnosis application. Thus, the data entered by the user will initially have the confidentiality level session enforced on it. Once condition appEnd is satisfied, the confidentiality level $\top$ must be enforced, implying that the data will be removed completely from the system. End-to-end enforcement of the policies will ensure that information derived from the user's symptoms will have the same policy, session appEnd/ $T$ enforced on it, or something more restrictive. Thus, any diagnoses derived from the user's symptoms must also have the confidentiality level $T$ enforced on them once appEnd is satisfied.

Condition satisfaction determines when policies mandate erasure. Since condition satisfaction is determined solely by the system state, we say that policy $p$ requires information erasure in state $s$ (or simply, requires erasure in state $s)$, denoted reqErase $(p, s)$, if there is a currently enforced erasure policy whose condition is satisfied. Figure 2 gives inference rules defining reqErase $(p, s)$. Lattice policy $\ell$ never requires erasure. Declassification policy $p \searrow^{a} q$ requires erasure if subpolicy $p$ (the policy currently enforced) requires erasure. Erasure policy $p a / q$ requires erasure if subpolicy $p$ requires erasure, or $a$ is satisfied. If policy $p$ is enforced on information, then we must ensure that in any state $s$ such that $p$ requires erasure in $s$, the information either is removed from the system, or has a suitably restrictive policy enforced on it.

\subsection{Semantics}

Intuitively, the policies describe how the confidentiality of information may and must change as the system executes. We formalize this intuition by providing a semantics for policies.

The semantics of policy $p$ in state $s$, denoted $\llbracket p \rrbracket_{s}$, is a set of pairs of system states and confidentiality levels that describes what confidentiality levels may be enforced on information labeled $p$ in state $s$ as the system evolves from state $s$. If policy $p$ is enforced on information in state $s$, and $\left(s^{\prime}, \ell^{\prime}\right) \in \llbracket p \rrbracket_{s}$, then by the time the system reaches state $s^{\prime}$ (in zero or more steps), confidentiality level $\ell^{\prime}$ may be enforced on the information.

$$
\begin{aligned}
\llbracket \ell \rrbracket_{s}=\left\{\left(s^{\prime}, \ell^{\prime}\right) \mid s \rightarrow^{*} s^{\prime} \text { and } \ell \sqsubseteq \ell^{\prime}\right\} \\
\llbracket p \searrow^{a} q \rrbracket_{s}=\llbracket p \rrbracket_{s} \cup \bigcup\left\{\llbracket q \rrbracket_{s^{\prime}} \mid s \rightarrow^{*} s^{\prime} \text { and } s^{\prime} \vDash a\right\} \\
\llbracket p a \nearrow q \rrbracket_{s}=\llbracket p \rrbracket_{s} \cap\left(\left\{\left(s^{\prime}, \ell\right) \in \llbracket p \rrbracket_{s} \mid\left[s, s^{\prime}\right] \not \models a\right\} \cup\right. \\
\left.\bigcup\left\{\llbracket q \rrbracket_{s^{\prime \prime}} \mid s \rightarrow^{*} s^{\prime \prime} \text { and }\left[s, s^{\prime \prime}\right) \not \models a\right\}\right)
\end{aligned}
$$

Figure 3. Semantics for policies $\llbracket p \rrbracket_{s}$

Figure 3 defines the semantics $\llbracket p \rrbracket_{s}$. We assume that the relation $\rightarrow$ over system states describes atomic transitions of the system, and denote the reflexive transitive closure of this relation as $\rightarrow^{*}$.

The semantics for confidentiality level $\ell$ allow any confidentiality level at least as restrictive as $\ell$ to be enforced at all times in the future.

The semantics of declassification policy $p \searrow^{a} q$ is a superset of the semantics of policy $p$. The semantics capture the intuition that when the condition is satisfied, the information may be declassified, and after declassification, policy $q$ is enforced on the declassified information. If $p$ permits enforcing confidentiality $\ell$ in state $s^{\prime}$, then $p \searrow^{a} q$ also permits it, and in addition, permits policy $q$ to be enforced on information, starting in any state $s^{\prime}$ such that $s^{\prime} \vDash a$.

By contrast, the semantics of erasure policy $p^{a} / q$ in state $s$ is a subset of the semantics of $p$ in $s$. The intuition is that policy $p$ is enforced while condition $a$ is not satisfied, and once condition $a$ is satisfied, the information is made more restricted by enforcing both policies $p$ and $q$. We write $\left[s, s^{\prime}\right] \not \models a$, where $s \rightarrow^{*} s^{\prime}$, to mean that condition $a$ is not satisfied in any state from $s$ to $s^{\prime}$ inclusive:

$$
\left[s, s^{\prime}\right] \not \models a \triangleq \forall s^{\prime \prime} .\left(s \rightarrow^{*} s^{\prime \prime} \wedge s^{\prime \prime} \rightarrow^{*} s^{\prime}\right) \Rightarrow s^{\prime \prime} \not \models a .
$$

Similarly, we use $\left[s, s^{\prime}\right) \not \models a$, to mean that condition $a$ is not satisfied in any state from $s$ up to but not including, state $s^{\prime}$ :

$\left[s, s^{\prime}\right) \not \models a \triangleq \forall s^{\prime \prime} .\left(s \rightarrow^{*} s^{\prime \prime} \wedge s^{\prime \prime} \rightarrow^{*} s^{\prime} \wedge s^{\prime \prime} \neq s^{\prime}\right) \Rightarrow s^{\prime \prime} \not \models a$.

\subsection{Relabeling judgment}

We can define a relabeling judgment $a_{0}, \ldots, a_{k} \vdash p \leq q$ such that if $a_{0}, \ldots, a_{k} \vdash p \leq q$ then, assuming conditions $a_{0}, \ldots, a_{k}$ are all satisfied, information labeled with policy $p$ can safely be relabeled with policy $q$. That is, enforcing $q$ on the information is consistent with policy $p$. Any such relabeling judgment should be sound with respect to the semantics, and we require the following property to hold. 
Property 1 (Soundness) If $a_{0}, \ldots, a_{k} \vdash p \leq q$ then for all states $s$, such that $\forall i \in 0 . . k$. $s \vDash a_{i}$, we have $\llbracket q \rrbracket_{s} \subseteq \llbracket p \rrbracket_{s}$.

A sound relabeling judgment serves as a syntactic approximation of the policy semantics. Inference rules for a relabeling judgment, and a proof of soundness, are given in the companion technical report [3]. In the following section, we use the relabeling judgment in the type system to enforce policies syntactically, without reference to policy semantics.

Other sound syntactic approximations of the policy semantics are possible. In earlier work [2] we introduced a sound relabeling relation parameterized on the current state of the system. This permits reasoning about the subsequent execution of the system, in addition to the conditions satisfied in the current system state.

\section{Language}

In this section we present a simple imperative language, $\mathrm{IMP}_{E}$, that incorporates declassification and erasure policies. The language has runtime mechanisms for erasure and declassification, and a type system to control the flow of information. In Section 4, we show that these together suffice to enforce declassification and erasure policies.

\subsection{Syntax}

$\begin{array}{cc}e::= & \text { Expressions } \\ n & \text { Integer literal } \\ x & \text { Variable } \\ e_{0} \oplus e_{1} & \text { Binary operation } \\ c::= & \text { Commands } \\ \text { skip } & \text { No-op } \\ x:=e & \text { Assignment } \\ c_{0} ; c_{1} & \text { Sequence } \\ \text { if } e \text { then } c_{0} \text { else } c_{1} & \text { Selection } \\ \text { while } e \text { do } c & \text { Iteration } \\ x:=\operatorname{declassify}\left(e, p_{f} \text { to } p_{t} \text { using } e_{0}, \ldots, e_{k}\right) & \text { Guarded declassification }\end{array}$

\section{Figure 4. Syntax of $\mathrm{IMP}_{E}$}

Figure 4 presents the syntax of $\mathrm{IMP}_{E}$. We assume there is a countable set of variables Vars. Language expressions include integer literals $n \in \mathbb{Z}$, and variables $x \in$ Vars. The metavariable $\oplus$ ranges over total binary operations on integers.

Conditions of policies in $\mathrm{IMP}_{E}$ are simply expressions. A condition is satisfied when it evaluates to a non-zero value. For example, if policy $H \searrow^{x+3} L$ is enforced on information, that information may be declassified when expression $x+3$ is non-zero.

The commands are standard, with the exception of declassification. The guarded declassification command $x:=$ declassify $\left(e, p_{f}\right.$ to $p_{t}$ using $\left.e_{0}, \ldots, e_{k}\right)$ evaluates expression $e$, and assigns the result to variable $x$, provided that expression $e_{i}$ evaluates to a non-zero value, for all $0 \leq i \leq k$. If there is some $e_{i}$ that evaluates to zero, then declassification fails. The expressions $e_{i}$ are conditions that must be satisfied for the declassification to occur. The guarded declassification command allows the type system to check that, assuming all conditions $e_{i}$ are satisfied, information labeled $p_{f}$ can safely be relabeled $p_{t}$, and allows the operational semantics to ensure that conditions $e_{i}$ are indeed satisfied when declassification occurs. The type system and runtime mechanisms for enforcing declassification are discussed further in Sections 3.2 and 3.3.

\subsection{Operational semantics}

A memory $\sigma$ is a map from variables to integers, and is thus a function from Vars to $\mathbb{Z}$. We write $\sigma(e)$ for the result of evaluating expression $e$ using memory $\sigma$, that is, using $\sigma(x)$ as the value of each variable $x$ that occurs in $e$. We write $\sigma[x \mapsto v]$ for the memory that maps variable $x$ to integer $v$, and otherwise behaves exactly as $\sigma$ does.

A configuration is a pair of a command $c$ and memory $\sigma$, written $\langle c, \sigma\rangle$. A configuration fully describes the system state. Since policy conditions are expressions, the satisfaction of a condition depends only on the memory of the current configuration. For brevity, we thus write reqErase $(p, \sigma)$ instead of reqErase $(p,\langle c, \sigma\rangle)$.

We assume there is a typing context that indicates what policy should be enforced on information stored in each variable. A typing context $\Gamma$ is a function from Vars to policies, and $\Gamma(x)$ is the policy that must be enforced on information stored in variable $x$. The typing context does not change during execution: a variable $x$ always has the same policy $\Gamma(x)$ enforced on it.

Figure 5 presents the operational semantics for $\mathrm{IMP}_{E}$, showing how configurations are updated as commands execute. The enforcement of policies relies on two runtime mechanisms, embodied in the operational semantics. The first is runtime overwriting of variables to enforce erasure; the second is runtime checking of conditions for declassification. Except for these two mechanisms, the operational semantics of the language are standard.

Overwriting variables. $\mathrm{IMP}_{E}$ enforces erasure by setting the contents of a variable to zero whenever the policy for the variable requires information erasure. Policy $p$ requires information erasure when reqErase $(p, \sigma)$ holds, 
OS-SKIP

$$
\langle\mathbf{s k i p} ; c, \sigma\rangle \rightarrow\langle c, \sigma\rangle
$$

OS-ASSIGN

$\frac{\sigma^{\prime}=\text { update }(\sigma, x, \sigma(e))}{\langle x:=e, \sigma\rangle \rightarrow\left\langle\text { skip }, \sigma^{\prime}\right\rangle}$
OS-SEQUENCE

$\frac{\left\langle c_{0}, \sigma\right\rangle \rightarrow\left\langle c_{0}^{\prime}, \sigma^{\prime}\right\rangle}{\left\langle c_{0} ; c_{1}, \sigma\right\rangle \rightarrow\left\langle c_{0}^{\prime} ; c_{1}, \sigma^{\prime}\right\rangle}$
OS-IF

$$
\frac{i= \begin{cases}0 & \text { if } \sigma(e) \neq 0 \\ 1 & \text { if } \sigma(e)=0\end{cases} }{\left\langle\text { if } e \text { then } c_{0} \text { else } c_{1}, \sigma\right\rangle \rightarrow\left\langle c_{i}, \sigma\right\rangle}
$$

OS-WHILE

$\langle$ while $e$ do $c, \sigma\rangle \rightarrow\langle$ if $e$ then $c$; while $e$ do $c$ else skip, $\sigma\rangle$

$$
\begin{aligned}
& \text { OS-DECLASSIFY } \\
& v=\left\{\begin{array}{ll}
\sigma(e) & \text { if } \forall i \in 0 . . k . \sigma\left(e_{i}\right) \neq 0 \\
0 & \text { if } \exists i \in 0 . . k . \sigma\left(e_{i}\right)=0
\end{array} \quad \sigma^{\prime}=\operatorname{update}(\sigma, x, v)\right. \\
& \left\langle x:=\operatorname{declassify}\left(e, p_{f} \text { to } p_{t} \text { using } e_{0}, \ldots, e_{k}\right), \sigma\right\rangle \rightarrow\left\langle\text { skip, } \sigma^{\prime}\right\rangle
\end{aligned}
$$

\section{Figure 5. Operational semantics of $\mathrm{IMP}_{E}$}

$$
\begin{aligned}
\operatorname{update}(\sigma, x, v) & =\left\{\begin{array}{l}
\operatorname{erasure}(\sigma) \\
\operatorname{erasure}(\sigma[x \mapsto v])
\end{array}\right. \\
\operatorname{erasure}(\sigma) & =\bigsqcup_{i \in \omega} \sigma_{i}
\end{aligned}
$$

where $\sigma_{0}=\sigma$, and

$$
\sigma_{i+1}=\lambda x \in \text { Vars. } \begin{cases}0 & \text { if reqErase }\left(\Gamma(x), \sigma_{i}\right) \\ \sigma_{i}(x) & \text { otherwise }\end{cases}
$$

and $\bigsqcup_{i \in \omega} \sigma_{i}$ denotes the least upper bound of the chain $\sigma_{0} \sigma_{1} \sigma_{2} \ldots$ under the ordering $\sqsubseteq$, where

$\sigma^{\prime} \sqsubseteq \sigma^{\prime \prime} \triangleq \forall x \in$ Vars. $\sigma^{\prime}(x)=\sigma^{\prime \prime}(x) \vee \sigma^{\prime \prime}(x)=0$

Figure 6. update $(\sigma, x, v)$ and $\operatorname{erasure}(\sigma)$

where $\sigma$ is the current memory. For example, policies $L^{x \geq 0} / H$ and $\left(L^{x=3} / H\right) \backslash y L$ both require information erasure if $\sigma(x)=3$. Since conditions are expressions, a condition may become satisfied when the memory is updated. The operational semantics for commands that update memory (assignment and declassification) use the utility function update $(\sigma, x, v)$ to overwrite variables, defined in Figure 6. The function update $(\sigma, x, v)$ takes memory $\sigma$, variable $x$, and integer $v$, and, provided policy $\Gamma(x)$ does not require erasure, returns erasure $(\sigma[x \mapsto v])$. The utility function erasure $(\sigma)$ checks for each variable $y$ if policy $\Gamma(y)$ requires erasure given the memory $\sigma$; if so, it overwrites variable $y$ with the value zero. Overwriting $y$ changes the memory, and thus may trigger the overwriting of other variables.

The function $\operatorname{erasure}(\sigma)$ is defined for all memories $\sigma$, and it provably overwrites variables as required: if $\sigma^{\prime}=$ erasure $(\sigma)$ then for all variables $x$, reqErase $\left(\Gamma(x), \sigma^{\prime}\right)$ implies $\sigma^{\prime}(x)=0$.

Runtime mechanism for declassification. Declassification of information can occur only when appropriate conditions are satisfied. For example, policy $H \searrow^{x>0} L$ allows information to be declassified to $L$ when the expression $x>0$ is non-zero, that is, when $x$ is positive. The operational semantics for a guarded declassification command, $x:=\operatorname{declassify}\left(e, p_{f}\right.$ to $p_{t}$ using $\left.e_{0}, \ldots, e_{k}\right)$, evaluates $e$ and assigns the result to variable $x$ provided the expressions $e_{0}, \ldots, e_{k}$ all evaluate to non-zero values. If one or more expressions $e_{i}$ evaluate to zero, then declassification fails, and variable $x$ is updated with the constant value zero. (Other reasonable semantics include leaving the value of $x$ unchanged, or stopping execution.)

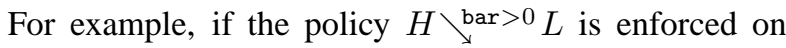
variable foo, then the command

$$
\text { quux := declassify }\left(\text { foo, } H \searrow^{\text {bar }>0} L \text { to } L \text { using bar }>0\right)
$$

will successfully declassify the contents of foo only if the expression bar $>0$ evaluates to a non-zero value. This

The use of runtime mechanisms to aid in the enforcement of declassification and erasure policies allows simpler static enforcement mechanisms. The policies can be enforced without these runtime mechanisms, but would require either more complex static enforcement, or less expressive conditions. See Section 7 for more discussion on this trade-off. 


\subsection{Type system}

The runtime mechanisms of $\mathrm{IMP}_{E}$ ensure that declassification only occurs if appropriate conditions are satisfied, and that variables are overwritten when their policies require erasure. However, the runtime mechanisms alone are not sufficient to ensure that erasure and declassification policies are enforced. What prevents information with erasure policy $L$ a $H$ from being stored in a variable $x$ that has policy $L$ enforced on it? Information in variable $x$ has low security enforced on it, and is not necessarily overwritten when condition $a$ is satisfied. Similarly, what prevents information with policy $H$ from being stored in a variable with policy $H \searrow^{a} L$ enforced on it, and subsequently (and incorrectly) declassified?

The type system of $\mathrm{IMP}_{E}$ restricts information flow within a program, ensuring that appropriate policies are enforced on information at all times. The type system restricts both explicit flows, where information flows from direct assignments to variables, and implicit flows [5], where information flows via the program's control structure. The type system does not restrict timing or termination channels.

The typing judgment $p c, \Gamma \vdash c$ com means that command $c$ is well-typed under typing context $\Gamma$ and program counter policy $p c$. The program counter policy is used to restrict implicit flows. It is an upper bound on the policies of information that may have influenced the value of the program counter, and so is an upper bound on the information that may be gained by knowing that command $c$ is executed. The typing judgment $\Gamma \vdash e: p$ exp means that under typing context $\Gamma$, policy $p$ is an upper bound on the policies of information that may be gained by evaluating expression $e$.

Figure 7 presents inference rules for these typing judgments. The rules track and restrict the flow of information within a program. For example, the rule T-ASSIGN for an assignment $x:=e$ ensures that information that may be revealed by evaluating expression $e$ is allowed to flow to variable $x\left(\vdash p_{e} \leq \Gamma(x)\right)$, and that information that may be revealed by learning the assignment is executed is also allowed to flow to variable $x(\vdash p c \leq \Gamma(x))$.

All the inference rules for the judgments $p c, \Gamma \vdash c$ com and $\Gamma \vdash e: p$ exp are standard for information-flow security type systems, with the exception of the rule for guarded declassification, T-DECLASSIFY. A guarded declassification command $x:=\operatorname{declassify}\left(e, p_{f}\right.$ to $p_{t}$ using $\left.e_{0}, \ldots, e_{k}\right)$ declassifies information with policy $p_{f}$ to policy $p_{t}$. Rule T-DECLASSIFY requires that $p_{f}$ can be relabeled $p_{t}$ assuming conditions conditions $e_{0}, \ldots, e_{k}$ are satisfied $\left(a_{0}, \ldots, a_{k} \vdash e_{0}, \ldots, e_{k} \leq p_{f} p_{t}\right)$. Rule T-DECLASSIFY also requires that the declassified information is allowed to be stored in $x\left(\vdash p_{t} \leq \Gamma(x)\right)$, that the information gained by knowing the declassification occurred can flow to $x(\vdash p c \leq \Gamma(x))$, and that the information gained by evalu- ating $e$ is bounded above by policy $p_{f}\left(\Gamma \vdash e: p_{f} \exp \right)$.

There is a flow of information from the conditions $e_{0}, \ldots, e_{k}$ to the variable $x$. The operational semantics for a guarded declassification will assign the result of evaluating $e$ into $x$ only if all conditions $e_{0}, \ldots, e_{k}$ evaluate to non-zero values. Thus, the value of the variable $x$ after the declassification command may reveal information about the value of the conditions. The typing rule for declassification, T-DECLASSIFY, tracks this information flow by requiring $\Gamma(x)$ to be an upper bound on the information that may be gained by knowing if condition $e_{i}$ was satisfied $\left(\Gamma \vdash e_{i}: \Gamma(x) \exp \right)$.

\subsubsection{Well-formed contexts}

A variable $x$ is overwritten when $\Gamma(x)$, the policy enforced on $x$, requires erasure. Thus, if satisfaction of condition $e$ can cause policy $\Gamma(x)$ to require erasure, there is information flow from $e$ to $x$. To track and control this information flow, we restrict the typing contexts that may be used.

For all variables $x$, we require that policy $\Gamma(x)$ is welltyped, written $\Gamma \vdash \Gamma(x)$ pol. Any policy that is used as a program counter policy in the proof of a typing judgment $p c, \Gamma \vdash c$ com must also be well-typed. The inference rule for well-typed policies is given in Figure 7. It requires that if condition $e$ may cause policy $p$ to require erasure, then $p$ is an upper bound on the information that may be obtained by evaluating $e$ ( $\Gamma \vdash e: p$ exp).

The recursively defined function eraseConds $(p)$ returns the set of expressions that may cause policy $p$ to require erasure. That is, reqErase $(p, \sigma)$ if and only if there is some condition $e \in \operatorname{eraseConds}(p)$ such that $\sigma(e) \neq 0$.

In addition, typing contexts are restricted to prevent infinite chains of variables $x_{0}, x_{1}, \ldots$, such that the overwriting of variable $x_{i}$ depends on the value of variable $x_{i+1}$. For example, this restriction prevents a variable $x$ having policy $L^{x=0} / H$. This restriction makes it easier to track information flows that occur due to overwriting, and simplifies both security proofs and implementation of variable overwriting. We define the overwrite dependency relation $\prec_{\Gamma}$ over variables such that $x \prec_{\Gamma} y$ if changing the value of $x$ may cause policy $\Gamma(y)$ to require erasure. More formally, $x \prec_{\Gamma} y$ if there is an expression $e$ such that $e \in$ eraseConds $(\Gamma(y))$ and $x$ appears in $e$.

Definition 1 (Well-formed typing context) Typing context $\Gamma$ is well-formed if the overwrite dependency relation $\prec_{\Gamma}$ is well-founded and for all $x \in \operatorname{Vars}, \Gamma \vdash \Gamma(x)$ pol.

\subsection{Example}

Figure 8 shows a fragment of $\operatorname{IMP}_{E}$ code that could be used to process a client request to the medical information 


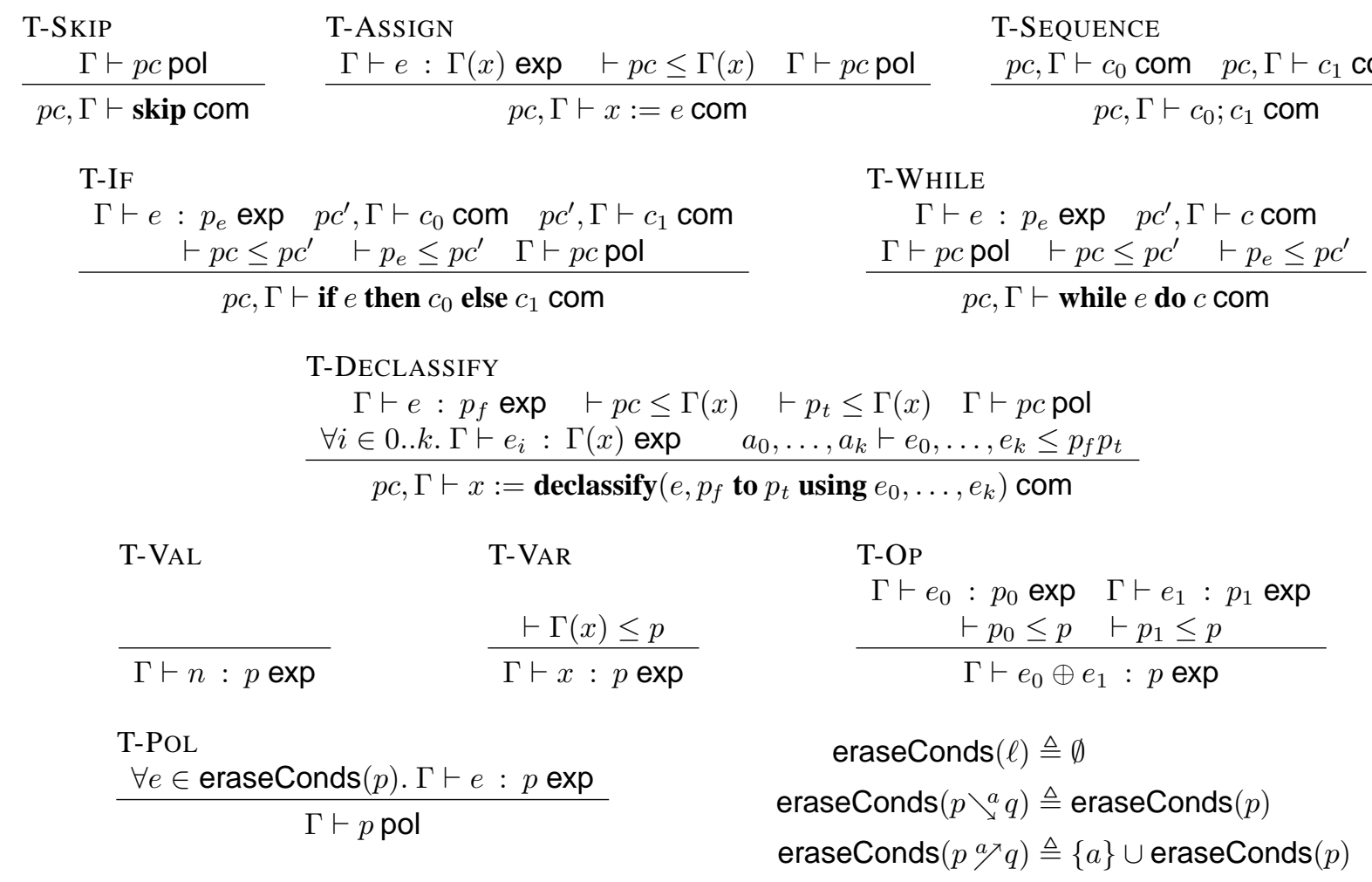

Figure 7. Inference rules for typing judgments $p c, \Gamma \vdash c \operatorname{com}, \Gamma \vdash e: p$ exp, and $\Gamma \vdash p$ pol

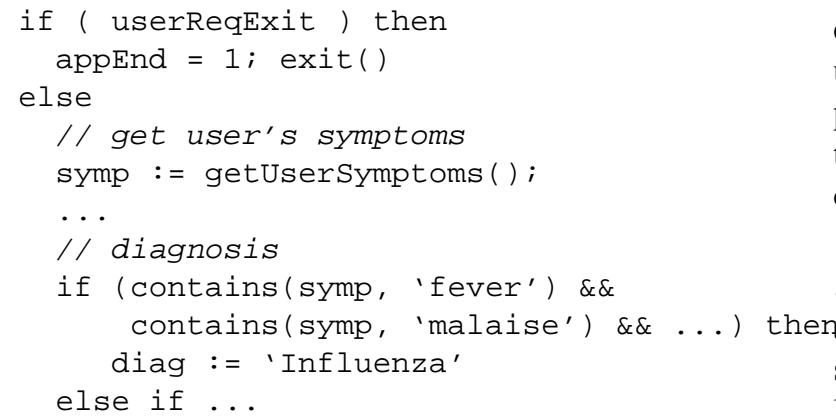

Figure 8. Medical information website example website described in the introduction. For ease of presentation, we assume the existence of functions and strings.

The code first checks if the user has requested to exit the diagnosis application, and if so, sets variable appEnd and exits. Otherwise, the code gets the user's symptoms and uses them to produce a diagnosis, which would then be displayed to the user. Modulo the use of strings and functions, the code is well-typed, and the relevant parts of the typing context $\Gamma$ are also shown in Figure 8.

The policy enforced on the user symptoms, $\Gamma$ (symp), is session appEndy T. As described in Section 2, session is a confidentiality level allowing only the session client and server to read the information, and $T$ is a confidentiality level so restrictive that it prevents the server from storing the information. There is an implicit flow of information from symp to diag, as symp is used in the conditional test on lines $8-9$, and diag is assigned to in the body of the conditional. By typing rule T-IF, the program counter policy for the conditional's body must be at least as restrictive as $\Gamma$ (symp). Similarly, by rule T-ASsign, $\Gamma$ (diag) must be as restrictive as that program counter policy. These constraints are satisfied by using policy $\Gamma$ (symp) as the program counter policy for the body of the conditional, since $\Gamma($ symp $)=\Gamma($ diag $)$.

The value of variable appEnd can cause policy 
session appEnd $\top$ to require erasure. Indeed, when variable appEnd is set (line 2), variables symp and diag are overwritten. There is thus information flow from appEnd to symp and diag. The requirement for a well-formed typing context tracks this flow, and requires that $\vdash \Gamma$ (appEnd) $\leq$ $\Gamma$ (symp) and $\vdash \Gamma$ (appEnd) $\leq \Gamma$ (diag), which are satisfied, as

$$
\begin{aligned}
\Gamma(\text { appEnd }) & =\text { session, } \\
\Gamma(\text { symp })=\Gamma(\text { diag }) & =\text { session } \text { appEnd }\rceil
\end{aligned}
$$

and

$$
\vdash \text { session } \leq \text { session } \text { appEnd } \top .
$$

\section{Security}

The type system and runtime mechanisms of $\mathrm{IMP}_{E}$ correctly enforce the security policies of Section 2.

\subsection{Noninterference}

Noninterference [7] is a well-known end-to-end semantic security condition which requires that secret inputs do not influence public outputs. A formal statement of noninterference depends on the definitions of secret input and public output. In this paper, we consider the secret input to be the contents of a single variable at the start of program execution, and the public output to be the values of some subset of variables during execution. To state noninterference formally, we define notions of observational equivalence of configurations, execution traces, and correspondences between traces.

The observation level of variable $x$ is determined by the policy $\Gamma(x)$ enforced on information stored in $x$. For policy $p, \operatorname{obs}(p) \in \mathcal{L}$ is the confidentiality level that is currently enforced on information labeled $p$, defined in Figure 9. The observation level of variable $x$ is obs $(\Gamma(x))$. Note that the observation level of a variable does not change during execution. The intuition is that a user with security clearance $\ell$ is only able to see the contents of variables with an observation level bounded above by $\ell$. For example, if variable $x$ has policy $\left(H \searrow^{a} L\right) b / H$ enforced on it, the observation level of $x$ is $H$, and a user with clearance $L$ could not observe the contents of $x$. The policy $\left(H \searrow^{a} L\right) b / H$ describes how the confidentiality of information stored in $x$ may and must change as conditions are satisfied, but does not change the observability of the variable itself.

Two configurations $\langle c, \sigma\rangle$ and $\left\langle c^{\prime}, \sigma^{\prime}\right\rangle$ are observationally equivalent at level $\ell$, written $\langle c, \sigma\rangle \approx_{\ell}\left\langle c^{\prime}, \sigma^{\prime}\right\rangle$, if all variables that are observable at level $\ell$ have the same value in both memories. Observational equivalence is implicitly parameterized on the typing context $\Gamma$. More formally, $\langle c, \sigma\rangle \approx_{\ell}\left\langle c^{\prime}, \sigma^{\prime}\right\rangle$ if and only if for all $x \in$ Vars,

$$
\begin{gathered}
\operatorname{obs}(\ell) \triangleq \ell \\
\operatorname{obs}\left(p \searrow^{a} q\right) \triangleq \operatorname{obs}(p) \\
\operatorname{obs}\left(p^{a} / q\right) \triangleq \operatorname{obs}(p)
\end{gathered}
$$

\section{Figure 9. Observation level}

if obs $(\Gamma(x)) \sqsubseteq \ell$ then $\sigma(x)=\sigma^{\prime}(x)$. Intuitively, if $\langle c, \sigma\rangle \approx_{\ell}\left\langle c^{\prime}, \sigma^{\prime}\right\rangle$, then a user with security clearance $\ell$ is unable to distinguish these two configurations by examining the contents of the memory. However, a user may be able to distinguish two executions of the program starting from $\langle c, \sigma\rangle$ and $\left\langle c^{\prime}, \sigma^{\prime}\right\rangle$, by observing the sequences of configurations that each execution produces. This motivates the definition of traces, and correspondences between traces.

A trace $\tau$ is a (finite or infinite) sequence of configurations $\tau=\left\langle c_{0}, \sigma_{0}\right\rangle\left\langle c_{1}, \sigma_{1}\right\rangle \ldots$ such that $\left\langle c_{i-1}, \sigma_{i-1}\right\rangle \rightarrow$ $\left\langle c_{i}, \sigma_{i}\right\rangle$ for all $i \in \mathbb{N}$ such that $0<i<|\tau|$, where $|\tau|$ denotes the length of trace $\tau$. We write $\tau[i]$ to refer to the $i$ th configuration in the trace $\tau$.

We use correspondences [1] between traces to indicate which states appear equivalent to an observer that sees first one trace, then the other. A correspondence $R$ is a relation over the natural numbers. If $R$ is a correspondence for traces $\tau_{1}$ and $\tau_{2}$, and $(i, j) \in R$, we will use it to mean that $\tau_{1}[i]$ and $\tau_{2}[j]$ look the same to a given observer. Formally, a correspondence $R$ between traces $\tau_{1}$ and $\tau_{2}$ is a subset of $\mathbb{N} \times \mathbb{N}$ such that

1. (Completeness) either $\{i \mid(i, j) \in R\}=\{i \in \mathbb{N} \mid i<$ $|\tau|\}$ or $\{j \mid(i, j) \in R\}=\left\{j \in \mathbb{N}|j<| \tau^{\prime} \mid\right\}$; and

2. (Initial configurations) if $|R|>0$ then $(0,0) \in R$; and

3. (Monotonicity) for all $(i, j) \in R$ and $\left(i^{\prime}, j^{\prime}\right) \in R$, if $i<i^{\prime}$ then $j \leq j^{\prime}$; and, symmetrically, if $j<j^{\prime}$ then $i \leq i^{\prime}$.

This definition ensures that a correspondence covers all configurations in at least one of $\tau$ or $\tau^{\prime}$, and if both traces are non-empty, then the initial configurations in the traces correspond to each other. The monotonicity requirement implies that the observer observes each trace as it executes, and time moves only forward.

Correspondences are both timing and termination insensitive, implicitly assuming that an observer cannot directly observe atomic transitions, and cannot detect if an execution has terminated. The definition can be refined to provide timing and/or termination sensitivity. Termination sensitivity is achieved by strengthening completeness to require that the correspondence covers all configurations in both $\tau$ and $\tau^{\prime}$, and that no configuration in $\tau$ or $\tau^{\prime}$ corresponds to an infinite set of configurations. Timing sensitivity is achieved by strengthening the definition so that every configuration in $\tau$ 
and $\tau^{\prime}$ corresponds to exactly one other configuration. Timing sensitivity implies an observer is able to observe each time step, and entails termination sensitivity.

Having defined traces, correspondences, and observational equivalence of configurations, we can now state noninterference. A command is noninterfering at level $\ell$ for variable $x$, if input supplied in the variable $x$ at the beginning of the program has no observable effect for a user with security clearance $\ell$, watching the execution of the system:

Definition 2 (Noninterference) A command $c$ with typing context $\Gamma$ is noninterfering at level $\ell$ for variable $x$ if for all integers $v_{1}, v_{2} \in \mathbb{Z}$, all memories $\sigma$, and all traces $\tau_{1}$ and $\tau_{2}$ such that $\tau_{i}[0]=\left\langle c\right.$, update $\left.\left(\sigma, x, v_{i}\right)\right\rangle$ for $i \in\{1,2\}$, there exists a correspondence $R$ for $\tau_{1}$ and $\tau_{2}$ such that for all $(i, j) \in R, \tau_{1}[i] \approx_{\ell} \tau_{2}[j]$.

The definition of noninterference relies on a typing context $\Gamma$, used in the definition of observational equivalence. For brevity, we omit mention of $\Gamma$ when clear from context.

Noninterference is too strong in the presence of declassification, which intentionally makes secret information public. Noninterference cannot express erasure requirements, which make publicly observable information less observable. Motivated by these shortcomings of noninterference, we defined noninterference according to policy.

\subsection{Noninterference according to policy}

Noninterference according to policy [2] is a semantic security condition that generalizes noninterference, and allows precise reasoning about the observability of information as it undergoes declassification and erasure.

Noninterference according to policy is defined in terms of the policy semantics, presented in Section 2. The intuition behind the policy semantics is that if information in state $s$ has policy $p$ enforced on it, then when the system enters state $s^{\prime}$, the information (or anything derived or influenced by it) should be observable at level $\ell$ only if $\left(s^{\prime}, \ell\right) \in \llbracket p \rrbracket_{s}$. Noninterference according to policy makes this intuition precise. Here, we specialize the definition of noninterference according to policy for $\mathrm{IMP}_{E}$ programs.

Definition 3 (Noninterference according to policy) $A$ command $c$ with typing context $\Gamma$ is noninterfering according to policy for variable $x$ if for all integers $v_{1}, v_{2} \in \mathbb{Z}$, all memories $\sigma$, memories $\sigma_{1}=\operatorname{update}\left(\sigma, x, v_{1}\right)$ and $\sigma_{2}=$ update $\left(\sigma, x, v_{2}\right)$, and all traces $\tau_{1}$ and $\tau_{2}$ such that $\tau_{i}[0]=\left\langle c, \sigma_{i}\right\rangle$ for $i \in\{1,2\}$, there exists a correspondence $R$ for $\tau_{1}$ and $\tau_{2}$ such that for all $(i, j) \in R$, for all $\ell \in \mathcal{L}$, if $\left(\tau_{1}[i], \ell\right) \notin \llbracket \Gamma(x) \rrbracket\left\langle c, \sigma_{1}\right\rangle$ and $\left(\tau_{2}[j], \ell\right) \notin \llbracket \Gamma(x) \rrbracket\left\langle c, \sigma_{2}\right\rangle$, then $\tau_{1}[i] \approx_{\ell} \tau_{2}[j]$.

Like noninterference, noninterference according to policy places restrictions on whether information input in variable $x$ is observable by a user during the execution of the program. However, whereas noninterference required all corresponding configurations to be observationally equivalent at a fixed level $\ell$, noninterference according to policy is more precise, and requires corresponding configurations to be observationally equivalent at confidentiality levels determined by the semantics of the policy enforced on the input. Thus, noninterference according to policy reflects how the observability of input may change during the execution of the system, as declassifications and erasures occur.

Noninterference according to policy generalizes noninterference. In particular, if the policy enforced on a variable $x$ indicates that information will never be observable at a confidentiality level $\ell$, then noninterference according to policy for variable $x$ implies noninterference at level $\ell$ for variable $x$. For example, a program that is noninterfering according to policy and takes input in variable $x$ with policy $H$ enforced on it, will never declassify the input to level $L$, and thus is noninterfering at level $L$ for $x$. The following theorem states this formally.

Theorem 1 For all commands c, typing contexts $\Gamma$, and variables $x$, if $c$ is noninterfering according to policy for variable $x$, then for all confidentiality levels $\ell$ such that for all memories $\sigma, \ell \notin\left\{\ell^{\prime} \mid\left(s, \ell^{\prime}\right) \in \llbracket \Gamma(x) \rrbracket_{\langle c, \sigma\rangle}\right\}$, c is noninterfering at level $\ell$ for variable $x$.

The central result of this paper is that the type system and runtime mechanisms of $\mathrm{IMP}_{E}$ suffice to enforce erasure and declassification policies. Thus, any well-typed $\mathrm{IMP}_{E}$ program is noninterfering according to policy.

Theorem 2 For all typing contexts $\Gamma$ and commands $c$, if $\Gamma$ is well-formed, and $p c, \Gamma \vdash c \operatorname{com}$ for some policy $p c$, then for all variables $x \in$ Vars, $c$ is noninterfering according to policy for variable $x$.

The proof of Theorem 2 is given in the companion techinical report [3]. It uses Pottier and Simonet's noninterference proof technique [20].

\section{To Jif and beyond}

The Jif programming language [18] extends Java [8] with information-flow control, allowing security policy annotations on program variables and method signatures. In this section, we describe how we extended Jif with declassification and erasure policies, and mechanisms to enforce these policies. The resulting language is called $\mathrm{Jif}_{E}$.

\subsection{Decentralized label model}

Security policies in Jif are from the decentralized label model (DLM) [17]. In DLM labels, security principals declare confidentiality and integrity restrictions on information. The reader policy $o \rightarrow r$ means that the principal $o$ 
owns the policy, and allows principal $r$ to learn, or read, information; the writer policy $o \leftarrow w$ is also owned by principal $o$, who allows principal $w$ to influence, or write, information. ${ }^{1}$ A label consists of conjunctions $(\sqcup)$ and disjunctions $(\sqcap)$ of reader and writer policies. Within a label, different principals may declare different restrictions, making the DLM suitable for reasoning about security in the presence of mutual distrust between principals. Variable types and method signatures in Jif may be annotated with labels. A labeled type is a pair of a base type (a primitive type or class) and a label.

We extended the DLM to allow principals to specify confidentiality restrictions using declassification and erasure policies. That is, declassification and erasure policies may now appear in reader policies on the right of the arrow.

The base lattice of confidentiality levels is the set of security principals, which is closed under conjunction $(\wedge)$ and disjunction $(\vee)[14,24]$, and so forms the necessary lattice structure. For example, the reader policy Alice $\rightarrow$ $(B o b \vee C h u c k) a \nearrow B o b$ is owned by Alice, who requires the

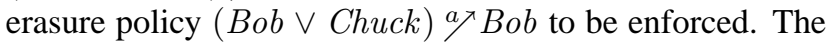
erasure policy initially allows Bob or Chuck to read information, but once $a$ is satisfied, only Bob may read it.

Instead of security principals, we could have used the decentralized labels as the base lattice. This would allow labels such as $($ Alice $\rightarrow$ Bob $) \backslash a($ Chuck $\rightarrow$ Dave $)$. However, this approach runs counter to the decentralized philosophy: different principals cannot declare different declassification and erasure requirements.

For the condition language, we allow a restricted class of expressions: access path expressions of type condition, and negations of these access path expressions. The type condition is a new primitive type with two values: true and false. Expressions of type condition may be cast to boolean, and vice versa. An access path expression is an expression of the form $r . f_{1} \ldots \ldots f_{n}$, where $r$ is a local variable, the special variable this, or a class name; each $f_{i}$ is a field; and all path elements other than the last are declared final. Immutability of path elements is needed for sound reasoning about conditions within the type system.

\subsection{Syntax and semantics}

$\mathrm{Jif}_{E}$ extends Jif's syntax and runtime system to incorporate the guarded declassification syntax and runtime erasure mechanisms of Section 3.

$\mathrm{Jif}_{E}$ contains the new guarded declassification expression $\operatorname{declassify}\left(e, L_{f}\right.$ to $L_{t}$ using $\left.e_{0}, \ldots, e_{k}\right)$, where $L_{f}$ and $L_{t}$ are labels, and each expression $e_{i}$

\footnotetext{
${ }^{1}$ The mnemonic for arrow direction in reader and writer policies is that in a reader policy $o \rightarrow r$, information may flow to principal $r$, whereas in a writer policy $o \leftarrow w$, information may flow from principal $w$.
}

is of type condition. The expression is evaluated by first evaluating $e$ to a value $v$, then evaluating each $e_{i}$ in turn; if any $e_{i}$ evaluates to false, then an UnsatisfiedConditionException is thrown; otherwise, the expression evaluates to $v$. If the evaluation of $e$ or any $e_{i}$ results in an exception, the declassification expression also results in the exception. As in the typing rule for declassification in Figure 7, type checking ensures that $L_{f}$ may be relabeled $L_{t}$ under the assumption that all conditions $e_{i}$ are satisfied.

Note that Jif already provides a mechanism for selective declassification [16, 15, 19], whereby a declassification that weakens or removes a policy owned by principal $o$ requires $o$ 's authority. By contrast, guarded declassification does not require the authority of any principal, since given a reader policy $o \rightarrow(p \searrow a q)$, the principal $o$ has already stated that information may be declassified when condition $a$ is satisfied.

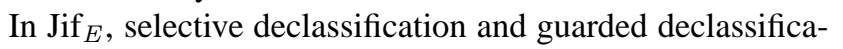
tion coexist as separate and independent mechanisms.

To enforce erasure policies, Jif $_{E}$ ensures that a variable or location that has label $L$ enforced on it is overwritten whenever any erasure policy in $L$ requires it. For example, if a location has the label $\{$ Alice $\rightarrow($ Bob this.f 7 Chuck $) \sqcap$ Dave $\rightarrow($ Alice this.o.d $\top \top)\}$ enforced on it, then the location is overwritten whenever either this. f or this.o.d evaluates to true. When a location or variable is overwritten, its contents are replaced with an appropriate default value. Thus, numeric locations are overwritten with zero, and reference locations are overwritten with null. Section 5.4 describes the runtime mechanisms used to achieve this. This erasure mechanism is analogous to the erasure mechanism of $\mathrm{IMP}_{E}$, which overwrites variables if the policy enforced on the variable requires erasure.

\subsubsection{Interaction with Java and Jif features}

Jif is intended for practical information-flow control. It supports a large subset of Java's language features, and provides additional features such as dynamic labels, constant arrays, and class and method polymorphism, needed for building real applications. The erasure enforcement mechanism of $\operatorname{IMP}_{E}$ needs careful adaptation for these language features.

Final fields and variables. In Java, fields, local variables, and formal arguments can be marked final, meaning their value will not change after initialization. To respect the finality of variables and locations, $\mathrm{Jif}_{E}$ requires that final variables and fields cannot be overwritten. The label $L$ enforced on a final field or variable must not contain any erasure policies, and if $L$ contains a dynamic label (see below), then the dynamic label must not contain any erasure policies. This ensures that label $L$ never requires erasure. 
Arrays. Jif allows different labels to be enforced on the elements of an array and the array itself. If the label enforced on the elements of an array requires erasure, the array is overwritten with appropriate default values; the length of the array is not altered. Jif supports constant arrays, whose elements cannot be modified after initialization. As with final fields, labels on elements of constant arrays must never require erasure.

Dynamic labels. Jif can represent labels at runtime and treat labels as first-class values. The primitive type label is the type of runtime labels, and Jif permits runtime comparisons of dynamic labels. $\mathrm{Jif}_{E}$ extends the runtime representation of labels to permit declassification and erasure policies to also be represented at runtime. We introduce a new kind of label, to reason about runtime labels that may require erasure. The primitive type elabel is used for dynamic labels that may require erasure. Only dynamic labels of type elabel may contain erasure policies; a dynamic label of type label cannot contain erasure policies. Thus, the labels of final fields, final variables, and elements of constant arrays, may refer to dynamic labels of type label, but may not refer to dynamic labels of type elabel. The type label can be cast to elabel, but not vice versa. The restriction that only elabels may contain erasure policies also simplifies backwards compatibility of $\mathrm{Jif}_{E}$ with Jif.

Polymorphism. Jif provides polymorphism for the labels of method arguments. For example, the method signature double $\{a\}$ sine(double $\{$ Alice $\rightarrow$ Bob $\}$ a) states that the label on the value returned is the same as the label of the actual argument a, which can be no more restrictive than $\{$ Alice $\rightarrow B o b\}$. In Jif method bodies, the label of a formal argument is a polymorphic label, representing the label of actual argument, and bounded above by the argument label specified in the signature. However, because actual arguments may require erasure during the method body execution, we need to know what label to enforce on formal arguments in the method body. Thus, in $\mathrm{Jif}_{E}$, method bodies assume that the label of a formal argument is simply the argument label bound specified in the signature. This is sound, but not as permissive as Jif, and effectively removes argument label polymorphism. However, it is not overly restrictive: we successfully implemented a remote voting system in 14,000 lines of Jif $E_{E}$ code, as discussed in Section 6.

Jif also supports polymorphic classes, permitting classes to be parameterized on labels and principals. ${ }^{2} \mathrm{Jif}_{E}$ extends the class parameters to allow parameters of type elabel.

\footnotetext{
${ }^{2}$ Jif as of version 3.1 does not support Java generics, another form of class parameterization for polymorphism.
}

\subsection{Information flow}

Jif's existing type-system tracks information flow. As discussed in Section 3, condition satisfaction can itself be used as a covert storage channel. Jif ${ }_{E}$ extends Jif's type system to soundly track this potential information flow.

Condition satisfaction affects whether the expression declassify $\left(e, L_{f}\right.$ to $L_{t}$ using $\left.e_{0}, \ldots, e_{k}\right)$ declassifies $e$ or throws an UnsatisfiedConditionException. Jif $E$ requires that the label of each $e_{i}$ is no more restrictive than label $L_{t}$.

Condition satisfaction may also cause variables and locations to be overwritten. Jif $_{E}$ tracks these information flows analogously to the $\mathrm{IMP}_{E}$ policy typing judgment $\Gamma \vdash p$ pol. Jif $_{E}$ requires that whenever a label $L$ is declared in a program, for any erasure policy $p e \gamma q$ that occurs in $L$, the label of expression $e$ must be no more restrictive than $L$. Jif ${ }_{E}$ also requires that if $1 \mathrm{bl}$ is a dynamic label that occurs in $L$, then the value 1 bl must be no more restrictive than $L$. So, if $e$ is a condition that appears in $1 \mathrm{bl}$, then the label of $e$ is no more restrictive than $1 \mathrm{bl}$, and thus no more restrictive than L.

\subsection{Translation}

The Jif compiler [18] is a source-to-source compiler, producing Java code as output. Jif programs rely on a small trusted runtime library, implemented in Java, that provides functionality such as runtime comparisons of labels. We extended the runtime library, and modified the source-tosource translation, to provide runtime support for erasure.

The key idea is that if a variable or location may need to be overwritten depending on the satisfaction of a condition $a$, then a listener is registered with condition $a$; the listener is notified whenever the value of $a$ changes, and the listener will overwrite the variable or location if necessary.

If a local variable may need to be overwritten, then the translation moves the local variable to the heap, to allow a condition listener to access it, and overwrite it as needed.

Assignments to fields and local variables are translated to check that the variable or field does not currently require erasure. The combination of condition listeners and assignment checks ensures that whenever the label enforced on the variable or location requires erasure, the variable or location will be zero or null as appropriate.

Overwriting a variable or location of type condition may trigger the overwriting of other variables and locations. To ensure that updating a condition does not cause an infinite cascade of listener invocations, the type system of Jif ${ }_{E}$ requires that for all conditions $a$, the value of $a$ cannot (directly or indirectly) control whether $a$ needs to be overwritten. This is analogous to ensuring that the overwrite dependency relation $\prec_{\Gamma}$ of Section 3 is well-founded. 


\section{Case study: Civitas}

Using $\mathrm{Jif}_{E}$, we implemented Civitas [4], a practical, secure, remote voting system. The use of declassification and erasure policies in the implementation of Civitas help ensure that the system's security requirements are satisfied. This section discusses the experience of using $\mathrm{Jif}_{E}$ to implement Civitas.

Civitas guarantees strong security properties in the presence of a strong adversary. The design of Civitas refines a cryptographic voting scheme by Juels, Catalano, and Jakobsson [13]. The entities involved in a Civitas election include an election supervisor, voters, and election authorities, which are mutually distrusting entities that collaborate to run an election. A Civitas election has several phases.

1. Setup. The electoral roll is established and shared keys are generated.

2. Registration. Voters retrieve credentials from election authorities.

3. Voting. Voters vote using their credentials.

4. Tabulation. Election authorities tabulate the election results.

More details of the design and security assurances of Civitas are available in a recent publication [4].

Civitas is implemented in 14,000 lines of $\mathrm{Jif}_{E}$ code, with about 8,000 additional lines of Java code to perform I/O and implement cryptographic operations. Declassification and erasure policies are used in four distinct places.

- Generation of a shared key by authorities. During setup, authorities engage in a protocol to generate a shared El Gamal key pair. Each authority generates a share of the key pair, and publishes a commitment to it. Each authority publishes its share of the public key, but only after all commitments are published.

The label $\left\{A_{i} \rightarrow A_{i} \backslash\right.$ allCommPosted $\left.\perp ; A_{i} \leftarrow A_{i}\right\}$ is used for authority $A_{i}$ 's public key share. The declassification policy requires that initially the information is readable only by election authority $A_{i}$, and may be declassified to be readable by everyone (represented by the bottom principal $\perp$ ) when condition allCommPosted is satisfied. Condition allCommPosted is a field of type condition. It is easy to check that this field is only updated once $A_{i}$ has successfully retrieved all key commitments. The writer policy $A_{i} \leftarrow A_{i}$ indicates that the key share was influenced only by $A_{i}$.

- Commit-reveal protocol by authorities. During tabulation, the authorities jointly generate random bits, and each authority must believe that the bits are random. Each authority selects random bits, and publishes a commitment to these bits. Once all commitments are published, each authority reveals its bits, which can be combined to form a sequence of bits that all authorities agree are random.

Similar to the key shares, the label $\left\{A_{i} \rightarrow\right.$ $A_{i} \backslash$ allBitsPosted $\left.\perp ; A_{i} \leftarrow A_{i}\right\}$ is used for authority $A_{i}$ 's random bits. Condition allBitsPosted is a field of type condition, and it is easy to check that this field is only updated once $A_{i}$ has been able to successfully retrieve all bit commitments.

- Management of credential shares by authorities. During registration, each authority generates a credential share for each voter. Each voter contacts each authority to retrieve his shares, combining them into a credential that can be used to vote. After delivering the share to the voter, the authority removes the share from the system. This helps ensure that the voter's anonymity is not violated should $A_{i}$ be subsequently compromised. Authority $A_{i}$ enforces the label $\left\{A_{i} \rightarrow\right.$ $\left(A_{i}{ }^{\text {delivered }} \top \mathrm{\top}\right) \backslash$ deliveryReq $\left.\perp ; A_{i} \leftarrow A_{i}\right\}$ on each voter credential share. Condition deliveryReq is satisfied when the voter has requested his credential share, and has authenticated himself to the authority. The satisfaction of this condition allows the declassification of the share. ${ }^{3}$ Any copies of the information that were not declassified must be erased when condition delivered is satisfied upon successful retrieval by the voter.

- Management of voter credential shares by voting clients. After voter $V_{j}$ has retrieved all credential shares from the authorities, he combines them into a single credential, which he then uses to vote, publishing it together with his ballot. After combining the shares, the voter deletes them, to remove any record of which authority provided which share.

The voter enforces on each credential share the label $\left\{V_{j} \rightarrow\left(V_{j}\right.\right.$ postCombined $\left.\nearrow \top\right) \backslash$ combined $\left.\perp\right\}$. Upon combining the shares into a credential, condition combined is satisfied, and the voter can declassify the credential to allow it to be published with his ballot. After combining shares, condition postCombined is satisfied, and undeclassified copies of the shares (or of information derived from them) are erased.

$\mathrm{Jif}_{E}$ allows complex declassification and erasure security requirements to be clearly and unambiguously declared on the data. In addition to stating what security must currently be enforced on the data, the policies limit how the data may

\footnotetext{
${ }^{3}$ Ideally, the declassification policy should allow the share to be readable only by the voter $V_{j}$ it is intended for. In the protocol between authority $A_{i}$ and $V_{j}$, each authenticates to the other, and they establish a shared key $k$; the credential share is sent to $V_{j}$ encrypted with $k$. The reasoning supported by the DLM is not powerful enough to determine that information encrypted with $k$ is readable only by $A_{i}$ and $V_{j}$. Extending it to reason about the subtleties of cryptography would allow a more precise declassification policy, but is largely orthogonal to this work.
} 
be used in the future. The information flow analysis ensures that uses of the data conform to the declared security policies. This provides additional assurance that the Civitas implementation is correct. The policy annotations serve as a form of documentation, making complex information security requirements visible in the code itself.

\section{Related work}

The most closely related work is that of Hunt and Sands [12]. Concurrently with this work, they consider the enforcement of simple erasure policies of the form $\ell / \ell^{\prime}$, where erasure is required at the end of a lexical scope. These policies are a restricted instantiation of the policy framework used here, where policies cannot be nested and the condition language is limited to specifying the end of lexical scopes. Using flow-sensitive typing contexts [11], Hunt and Sands present an elegant type system to enforce erasure policies; their system requires no runtime erasure mechanism.

Comparing our work to Hunt and Sands' highlights a tension between expressiveness of erasure conditions and ease of enforcement. Simpler condition languages are easier to reason about statically, and thus easier to enforce statically. Hunt and Sands' conditions are tied to lexical scopes, and it is straightforward to reason statically about when conditions are satisfied. By contrast, the condition language used in this work is program expressions: flexible, but difficult to reason about statically. Because it is difficult or impossible to know the value of an arbitrary expression at a given program point prior to execution, it is difficult to determine statically whether a policy will require erasure at that program point, and thus difficult to enforce erasure statically. Instead, we use a simple runtime mechanism to enforce erasure, an approach similar in spirit to hybrid type checking [6].

Although runtime mechanisms are used to help enforce erasure and declassification, information flow control in $\mathrm{IMP}_{E}$ is static, using a type system to track and restrict the flow of information. Starting with Volpano, Smith and Irvine [25], type systems have proven successful in providing information flow control without the overhead of representing security labels at runtime; many of these type systems are surveyed by Sabelfeld and Myers [21], and Sabelfeld and Sands [22] discuss some of the recent type systems that consider declassification.

Hansen and Probst [10] consider information flow security in Java Card bytecode, and identify the utility of erasure policies in providing security assurance. They consider "simple erasure policies" of the form $L$ end $7 H$, where end is a condition indicating the end of execution of the current program. They define a corresponding simple erasure security condition. They conjecture, but do not demonstrate, that simple erasure is straightforward to enforce.

Hansen and Probst [9] have also used erasure policies in secure dynamic program repartitioning. Secure program partitioning [26] is a technique to split data and code across a set of mutually distrusting hosts while guaranteeing security. Hansen and Probst consider repartitioning a program when the set of hosts changes dynamically, and use erasure policies to ensure that old copies of data are removed from the system when repartitioning occurs. Hansen and Probst do not describe how to enforce the erasure policies. Søndergaard's subsequent master's thesis [23] discusses the trusted runtime components required to enforce these erasure policies, but does not implement them.

Sabelfeld and Sands [22] consider different aspects of declassification, and propose four semantic principles for declassification, three of which are applicable to erasure. Noninterference according to policy satisfies semantic consistency and conservativity, but does not satisfy nonocclusion precisely because, as Hunt and Sands [12] point out, the policies address when, but not what, information is erased and declassified.

\section{Conclusion}

In this paper we have shown how to enforce erasure requirements end-to-end in language-based settings. Erasure requirements are specified in a flexible and powerful policy framework [2] that can also express declassification requirements. The policies express when information may be declassified, and when information must be erased.

We have proved that an information-flow control type system, in conjunction with a runtime mechanism for erasure, can enforce the erasure and declassification policies in $\mathrm{IMP}_{E}$, a simple imperative language. Well-typed $\mathrm{IMP}_{E}$ programs satisfy noninterference according to policy [2].

The end-to-end enforcement of erasure and declassification policies is also practical: we have extended the Jif programming language [18] with erasure and declassification policies and enforcement mechanisms, and used the resulting language to implement a secure remote voting system.

The ultimate goal of this work is to make it easy for programmers to write secure programs, and to have assurance that these programs are secure. This work, by providing provably secure enforcement of expressive erasure and declassification policies, brings us closer to that goal.

\section{Acknowledgments}

We thank Michael Clarkson for comments on an earlier draft, Fred Schneider for discussions on how best to present this material, and Eric Breck for suggesting the medical diagnosis application. 


\section{References}

[1] A. Banerjee, D. A. Naumann, and S. Rosenberg. Expressive declassification policies and modular static enforcement. Technical Report CS Report 2007-04, Stevens Institute of Technology, Nov. 2007.

[2] S. Chong and A. C. Myers. Language-based information erasure. In Proc. 18th IEEE Computer Security Foundations Workshop, pages 241-254, June 2005.

[3] S. Chong and A. C. Myers. End-to-end enforcement of erasure and declassification. Technical Report 2008-XXXX, Cornell University, Apr. 2008.

[4] M. R. Clarkson, S. Chong, and A. C. Myers. Civitas: A secure voting system. In Proc. IEEE Symposium on Security and Privacy, May 2008.

[5] D. E. Denning and P. J. Denning. Certification of programs for secure information flow. Comm. of the ACM, 20(7):504513, July 1977.

[6] C. Flanagan. Hybrid type checking. In Proc. 33rd ACM Symp. on Principles of Programming Languages (POPL), pages 245-256, 2006.

[7] J. A. Goguen and J. Meseguer. Security policies and security models. In Proc. IEEE Symposium on Security and Privacy, pages 11-20, Apr. 1982.

[8] J. Gosling, B. Joy, G. Steele, and G. Bracha. The Java Language Specification. Addison Wesley, 2nd edition, 2000. ISBN 0-201-31008-2.

[9] R. R. Hansen and C. W. Probst. Secure dynamic program repartitioning. In Proc. Nordic Workshop on Secure ITSystems, Oct. 2005.

[10] R. R. Hansen and C. W. Probst. Non-interference and erasure policies for Java Card bytecode. In Proc. 6th International Workshop on Issues in the Theory of Security, Mar. 2006.

[11] S. Hunt and D. Sands. On flow-sensitive security types. In Proc. 33rd ACM Symp. on Principles of Programming Languages (POPL), pages 79-90, 2006.

[12] S. Hunt and D. Sands. Just forget it - the semantics and enforcement of information erasure. In Proc. 17th European Symposium on Programming, Mar. 2008.

[13] A. Juels, D. Catalano, and M. Jakobsson. Coercion-resistant electronic elections. In Workshop on Privacy in the Electronic Society, pages 61-70, Nov. 2005.

[14] B. Lampson, M. Abadi, M. Burrows, and E. Wobber. Authentication in distributed systems: Theory and practice. In Proc. 13th ACM Symp. on Operating System Principles (SOSP), pages 165-182, October 1991. Operating System Review, 253(5).

[15] A. C. Myers. JFlow: Practical mostly-static information flow control. In Proc. 26th ACM Symp. on Principles of Programming Languages (POPL), pages 228-241, Jan. 1999.

[16] A. C. Myers and B. Liskov. A decentralized model for information flow control. In Proc. 17th ACM Symp. on Operating System Principles (SOSP), pages 129-142, 1997.

[17] A. C. Myers and B. Liskov. Protecting privacy using the decentralized label model. ACM Transactions on Software Engineering and Methodology, 9(4):410-442, Oct. 2000.
[18] A. C. Myers, L. Zheng, S. Zdancewic, S. Chong, and N. Nystrom. Jif: Java information flow. Software release, http: //www.cs. cornell. edu/jif, July 2001.

[19] F. Pottier and S. Conchon. Information flow inference for free. In Proc. 5th ACM SIGPLAN International Conference on Functional Programming (ICFP), pages 46-57, 2000.

[20] F. Pottier and V. Simonet. Information flow inference for ML. In Proc. 29th ACM Symp. on Principles of Programming Languages (POPL), pages 319-330, 2002.

[21] A. Sabelfeld and A. C. Myers. Language-based informationflow security. IEEE Journal on Selected Areas in Communications, 21(1):5-19, Jan. 2003.

[22] A. Sabelfeld and D. Sands. Dimensions and principles of declassification. In Proc. 18th IEEE Computer Security Foundations Workshop, pages 255-269, June 2005.

[23] D. Søndergaard. Secure program partitioning in dynamic networks. Master's thesis, Technical University of Denmark, 2006. IMM-M.Sc-2006-92.

[24] S. Tse and S. Zdancewic. Designing a security-typed language with certificate-based declassification. In Proc. 14th European Symposium on Programming, 2005.

[25] D. Volpano, G. Smith, and C. Irvine. A sound type system for secure flow analysis. Journal of Computer Security, 4(3):167-187, 1996.

[26] S. Zdancewic, L. Zheng, N. Nystrom, and A. C. Myers. Untrusted hosts and confidentiality: Secure program partitioning. In Proc. 18th ACM Symp. on Operating System Principles (SOSP), pages 1-14, Oct. 2001. 